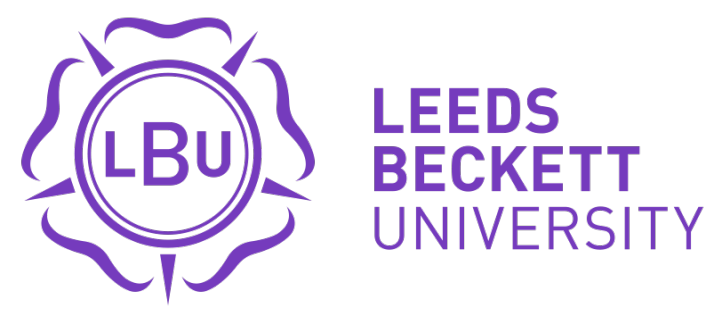

Citation:

Stevens, RC and Marchant, P and Raybould, D and Renshaw, T (2009) Are You Seeing What I'm Seeing? An Eye-Tracking Evaluation of Dynamic Scenes. Digital Creativity, 20 (3). pp. 153-163. ISSN 1462-6268 DOI: https://doi.org/10.1080/14626260903083611

Link to Leeds Beckett Repository record:

https://eprints.leedsbeckett.ac.uk/id/eprint/8402/

Document Version:

Article (Accepted Version)

Creative Commons: Attribution-Noncommercial 4.0

The aim of the Leeds Beckett Repository is to provide open access to our research, as required by funder policies and permitted by publishers and copyright law.

The Leeds Beckett repository holds a wide range of publications, each of which has been checked for copyright and the relevant embargo period has been applied by the Research Services team.

We operate on a standard take-down policy. If you are the author or publisher of an output and you would like it removed from the repository, please contact us and we will investigate on a case-by-case basis.

Each thesis in the repository has been cleared where necessary by the author for third party copyright. If you would like a thesis to be removed from the repository or believe there is an issue with copyright, please contact us on openaccess@leedsbeckett.ac.uk and we will investigate on a case-by-case basis. 


\title{
Are you seeing what I'm seeing? An eye-tracking evaluation of dynamic scenes
}

\author{
Paul Marchant, David Raybould, Tony Renshaw, Richard Stevens \\ Leeds Metropolitan University, Innovation North, Caedmon Hall, Headingley Campus, \\ Leeds. LS6 3QS \\ \{p.marchant, d.raybould, t.renshaw, \\ r.c.stevens\} @leedsmet.ac.uk
}

\begin{abstract}
Based on the data from the 2006/7 multimedia exhibition, 'Repossessed', during which over 400 members of the public watched scenes from Hitchcock's 'Vertigo', this paper describes the basis of an approach to the use of eye-tracking techniques, visualizations, and metrics to measure the influence of directorial techniques on film viewers' experience. Used as part of a repertoire of tools, the visualization and quantitative evaluation of eye movement data can provide an intuitive and accessible approach to the evaluation of moving image based media and allow the conventions, assumptions, and intuitive practices of film-making to be examined.
\end{abstract}

Keywords. Eye-tracking, evaluation, film theory and practice, directorial devices.

\section{Introduction and Aims}

The literature on film editing makes frequent references to the eyes as a vehicle for conveying emotion to the audience (Coan \& Allen, 2007) and assumptions about eye movement forms the basis of the majority of discussion on film production and editing technique (Dymtryk, 1984; Pepperman, 2004; Cutting, 2005). Whilst it is undoubtedly true that film-makers have a range of techniques through which they may attempt to impose an interpretation of an event (Bazin, 1967), film technique, derived largely from experience and intuition, assumes a homogeneity of audience reception, rather than an understanding that the audience may not only create their own meaning through their interaction with the film (Allen, 1990; Staiger, 1992) but may also actually physically view it in different ways.

Previous studies have shown that there is often a degree of common experience, where a range of viewers will focus their visual attention onto a common area (Itti, 2004; Vesterby, Voss, Hansen, Glenstrup, Hansen \& Rudolph, 2005; Goldstein, Woods \& Peli, 2007), however this seems highly dependent on the material being viewed and appears to be influenced by both low level sensory triggers such as motion and contrast (Stelmach, Tam \& Hearty, 1991; Findlay, 1984) and 'top down' conscious control mechanisms such as our attraction to the human face (Privitera \& Stark, 2000; Tosi, Mecacci \& Pasquali, 1997). There is as yet limited discussion re- 
garding the effects of cinematic devices deliberately engineered to control the gaze of the viewer.

If the traditional collective wisdom of film-making serves mostly as rules of thumb, though sometimes overruled by individual creators (Katz, 1991; Boorstin, 1991) then it is worth examining both the effectiveness of these rules and the impact of breaking conventions (D'Ydewalle \& Vanderbeeken, 1990; Smith, 2005).

The overt visual attention detected by an eye-tracker gives us a window on internal systems and has been used to evaluate learning, skill level and cognitive processing (Rayner, 1998; Duchowski, 2007; Webb \& Renshaw, 2008). Although work has been done in analysing the collective response to static scenes (Wooding, 2002) the study of visual perception of dynamic scenes is inherently more difficult as the objects that are being fixated upon are in constant motion. It is therefore difficult to relate the position of the fixation to any particular object or compare multiple viewing experiences without a painstaking visual analysis of the scene (Henderson \& Hollingworth, 1998).

This article examines two scenes from Alfred Hitchcock's Vertigo (Hitchcock, 1958). Using the data from a unique large scale study we have established methods for visualising multiple viewers' experience of a dynamic scene and for analysing the data statistically. Observed differences in eye movement characteristics, attributed to the director's shooting techniques, have been tested for statistical significance and the data further explored to determine whether there were significant gender related variations in eye movement patterns.

\section{Installation and Setup Description}

\subsection{The RePossessed Exhibition}

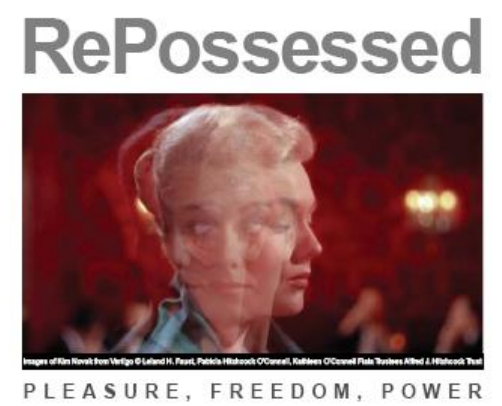

Fig. 1. RePossessed exhibition poster (Lane et al., 2007).

The RePossessed exhibition was installed at the Leeds Metropolitan University Gallery (24 October to 18 November 2006) and Waterman's Art Gallery, London (10 March to 22 April 2007) and was a collaboration between colleagues at London Metropolitan University and Leeds Metropolitan University.

The exhibits, based around Hitchcock's film Vertigo (Hitchcock, 1958), aimed to explore notions of freedom and power through the film's themes of voyeurism and surveillance and through an exploration of the relationships between author and audi- 
ence, both in terms of the film itself and within the context of new media and installation work.

In the piece 'ReViewed' over 400 members of the public watched excerpts from Vertigo whilst their eyes were tracked using a non-invasive Tobii (Tobii, 2006) eyetracker. After viewing the scene the participants could then log into a second booth. The system in this booth utilised a touch screen interface (Figure 2) and allowed the user to examine their own viewing experience of the film and compare it with up to seven other participants via a number of visualizations of the eye-tracking data.

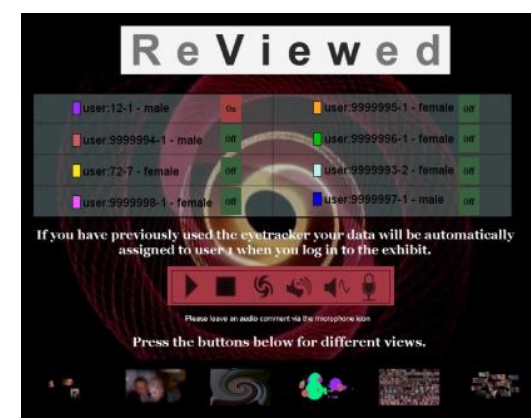

Fig. 2. The user interface for the ReViewed installation.

The 'Focus' visualization blurred out all the areas of the screen which were not being fixated upon (these were in sharp focus) (Figure 3); an overlay of coloured circles and squares (representing male and female respectively) allowed a comparison of gendered viewing strategies (Figure 4); and a collective summary visualization was produced that resized the parts of the film attended to according to how long the participants' gaze stayed in that location, providing an overview of their visual experience (Figure 5).

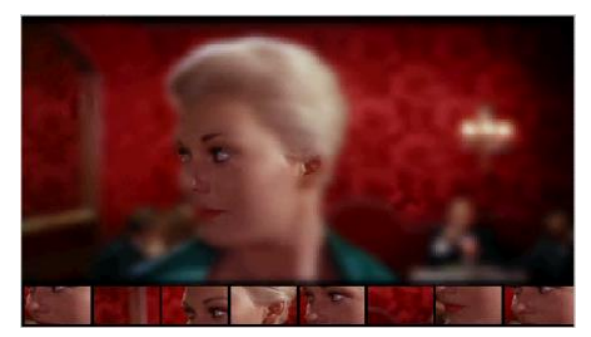

Fig. 3. Focus as visualization metaphor. This blurred out all the areas of the screen which were not fixated on. 


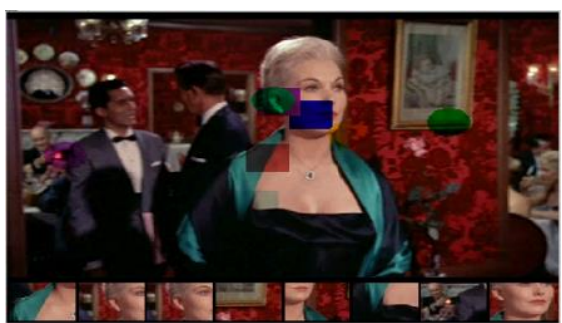

Fig. 4. Gender comparison visualization. An overlay of coloured circles and squares (representing male and female respectively) allowed a comparison of gendered viewing strategies.

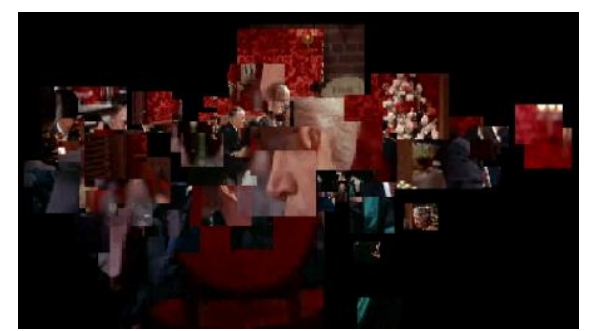

Fig. 5. Summary visualization. This resized the parts of the frame according to how long the participants' gaze stayed in that location.

During the exhibition it was only possible to visualize the eye-tracked fixation data of up to eight participants simultaneously. In this paper we outline our subsequent work in developing a visualization method for viewing an unlimited number of eye-tracking data sets simultaneously and the statistical method which allows a quantitative study of this data.

\subsection{Data Collection}

The eye-tracking system used a Tobii T1750 eye-tracking monitor (Tobii, 2006) and a Shuttle PC computer running the Clearview 2.7.1 (Clearview, 2006) imageprocessing and eye-tracking software. Participants were seated with the monitor positioned at eye level and then asked by a gallery facilitator to complete a calibration exercise before viewing one of two scenes selected from Vertigo. Two scenes from different parts of the film were used across the duration of the exhibit, to allow comparison of a variety of editing and directorial techniques.

In both locations the eye-tracker was set up with no peripheral visual distractions while extraneous noise was minimised using high quality closed headphones. All participants were monitored by a facilitator and any interrupted viewings, or viewings where the data showed a null record of gaze data (indicating that the participant was looking away from the screen) have not formed part of the final data set.

The eye-tracking system measures point-of-regard through the use of the 'cornealreflection/pupil centre' method (Goldberg \& Wichansky, 2003; Duchowksi, 2007). 
The raw data recorded by the eye-tracker captures the participant's eye gaze positions every $50^{\text {th }}$ of a second. The supplied software, Clearview, can identify fixations, where the eye dwells within a location for a defined length of time, separating them out from saccades (rapid eye movements between fixations) and blinks.

\section{Development of Software for Presentation of the Visualizations}

\subsection{The 'Heat-Maps'}

In eye-tracking, a 'heat-map' is a commonly used graphical representation of gaze data in which areas of an image with high concentrations of fixations are coloured red, while lower concentrations appear in cooler colours (Wooding, 2002). In our case, we used the 'heat-map' not for individuals' data but to show the degree of common experience, or commonality (the number of people looking in a similar location) within the scenes.

Our heat maps were made using a custom-built application created with the objectoriented programming language MaxMSP 5 (MaxMSP, 2008). In a four stage process, this combined all the visitor data into a single file, generated a grey-scale image for each frame, coloured these according to the convention just described, and finally overlaid them onto each frame of the original film clip (Figure 6).

A number of issues were addressed in the design of the application, including the difference between the frame-rate of the film and the sampling rate of the eye-tracker, eliminating errors in the data, and choosing how exactly to indicate fixations. For this last purpose we created a set of concentric circles fading from very dark grey at the centre to black at the perimeter, which were centred on the location of each fixation. As these build up through the accumulation of more and more individuals' samples, the areas with the most fixations become progressively lighter. Filtering on the finished heat-map image for each frame removes low values which are not needed (Figure 7).

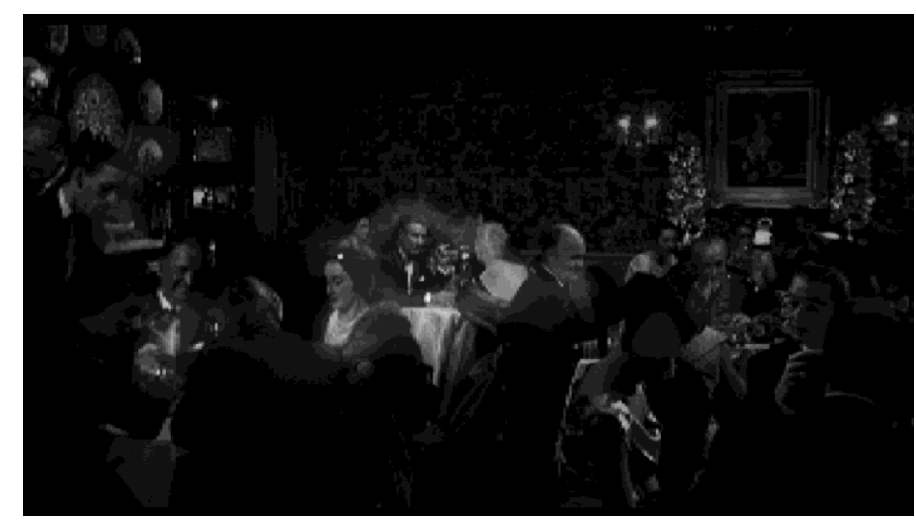

Fig. 6. A heat-map overlaid on top of original video. 


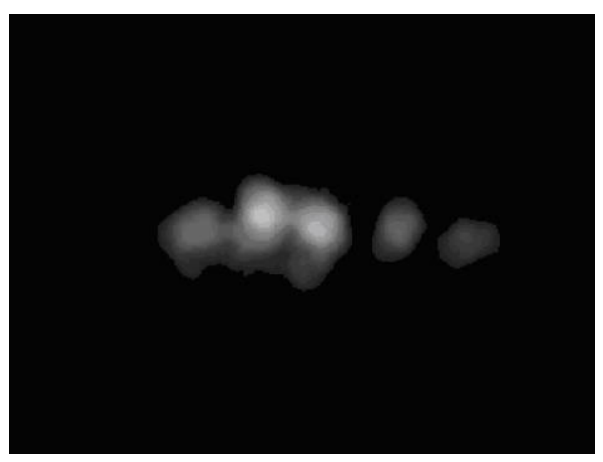

Fig. 7. A greyscale 'heat-map' after filtering out of low-level values.

Following the removal of any participants data which was incomplete as a result of an interrupted viewing, the following visualization analysis is based on 238 participants for the 'Ernies' scene, and 131 for the 'Apartment' scene.

\section{Visualization Analysis}

\subsection{The Director as the Controller of Attention}

Both as a man and as a director, Hitchcock is perhaps most associated with a reputation as a control freak (Spoto, 1983):

You know the public always likes to be one jump ahead of the story; they like to feel they know what's coming next. So you deliberately play upon this fact to control their thoughts. ...Psycho has a very interesting construction and that game with the audience was fascinating. I was directing the viewers. You might say I was playing them, like an organ. Hitchcock quoted in Truffaut (1984, p.73)

Part of Hitchcock's self-mythologizing has promoted the idea of Hitchcock as auteur whose films are the product of a very personal vision (Haeffner, 2005), even though the storyboards which purport to show the precise pre-conception of every shot in his films tend to obscure collaborations and compromises (Spoto, 1983). Kohn (2000) presents strong evidence that at least some were produced for publicity purposes after filming was over. Nevertheless, the use of visualizations such as ours provides an opportunity to examine how Hitchcock exerts control over the watching of his films through his use of cinematic techniques to manipulate the gaze.

\subsection{Strategies}

\subsubsection{Focus}

In the first scene the detective 'Scottie' (played by James Stewart) has been hired to spy on an old school friend's wife for her own protection. He is invited to observe 
them whilst they have a meal in 'Ernies' restaurant. This shot is filmed in deep focus and so all three planes - foreground, mid-ground and background - are uniformly sharp (Figure 8). Film theorists such as André Bazin have argued that the use of long deep focus shots provides a more objective democratic reality in that it allows the audience to interpret the scene for themselves in contrast to the 'coercion' of montage (Bazin, 1967).

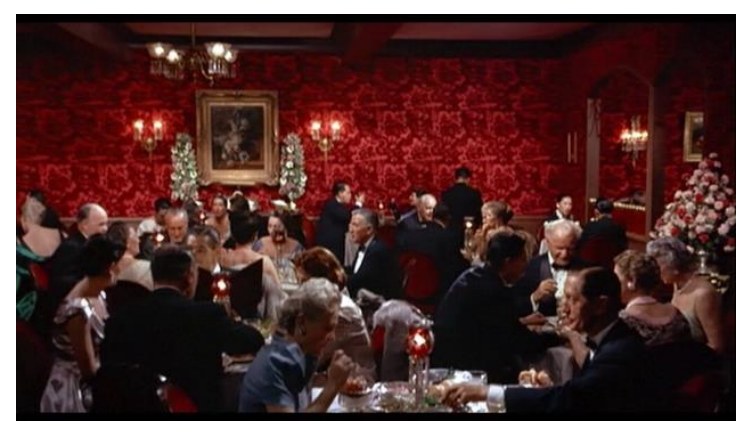

Fig. 8. Deep focus shot in 'Ernies' restaurant scene.

As the scene opens we can see how the close view and central framing of the main protagonist results in a concentration of the audience's visual attention.

\subsubsection{Other Cinematic Devices}

As Scottie casts his eyes across the restaurant, the camera pulls back and moves laterally across the restaurant scene. According to Bazin's theory (Bazin, 1967), the use of deep focus in this shot should result in a disparate range of points of visual attention. Whilst this was partly borne our in our visualizations by the resultant dynamic heatmap, the combined effect of the audience seeking the aim of the character's gaze and of the leftward momentum of the camera produces a bias towards the left hand side of the frame. It is clear that the director has succeeded in directing the viewer's attention to a significant extent.

From frame 672, the camera stops its leftwards momentum and moves forward, bringing two other characters (Madeleine and her husband) central to the frame. In addition to the visual contrast of Madeleine's emerald green sash standing out in contrast to the muted black, white, and grey of the other diners, the camera movement and central framing are again effective in their attentional draw.

Around frame 458, there is an exception to the generally disparate viewing behaviour in relation to this sequence that is worthy of comment. Previous work has noted how change per se, and particularly motion, attract visual attention (Stelmach et al., 1991; Findlay, 1984). Although not universally attended to, the movement and contrasted colour of a background character drinking a toast clearly formed a focal point at this point in the sequence. 


\subsection{Controlling Attention Between Shots and Across Edits}

The second half of the scene has a different character that appears effective in enforcing an even greater control of the viewer's visual attention.

Strong similarities between compositional elements from one shot to the next (graphic matches) - in similar positions, shapes, colours, etc. are sometimes used to create allusions and associations (Bordwell \& Thompson, 2007). Even when this is not the case, the need for the viewer to track the object of interest across the screen in an auditorium, possibly even having to turn their head, must have been a consideration in the development of this aspect of continuity editing.

Following this principle, the framing and positioning of the central characters from shot to shot is remarkably consistent. Additionally, as Madeleine approaches Scottie, a selective, shallow focus strengthens this hold still further as the background diners disappear into blur.

Later we again see how consistency of relationships, within the 'rules' of continuity editing, has the effect of holding the viewers' gaze on certain areas of the screen. The $180^{\circ}$ rule states that the camera must stay on one side of the actions and objects in a scene (Bordwell \& Thompson, 2007), ensuring that the shot will have consistent spatial relations and screen directions. In an initial over-the-shoulder two-shot set up of the 'Apartment' scene, the physical location of each character is maintained within the frame. Later in the same scene the graphic continuity between shots becomes even more pronounced through the careful planning of compositional elements.

Anchored by the consistent position of a lamp and precise matching of position, there is very little deviation from a common area of focused visual attention during the five edits and 16 seconds that make up the subsequent sequence (Figure 9).
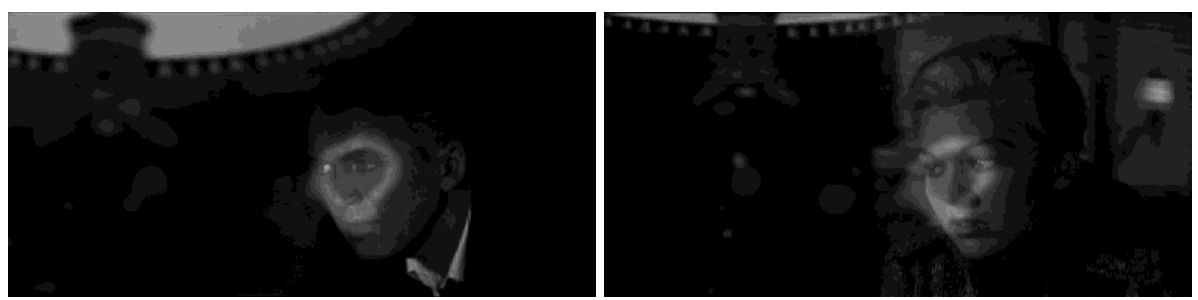

Fig. 9. 'Apartment' scene; frames 3142 (left) and 3143 (right).

\subsection{Summary of Visualization Analysis}

Our analysis serves to demonstrate the potential for the dynamic visualization techniques we have developed as an evaluative tool. In this short sequence we have noted the effect of these strategies and techniques on the audience's visual attention:

- Focus

- Camera movement

- Eye line match

- Colour and contrast

- Motion of elements within the shot 
- Graphic matching and framing

Although useful, our visualizations are to some extent still open to interpretation (relying on the hue of the colour to indicate the number of active viewers, for example). In order to provide more quantitative evidence a statistical method has also been developed and is explained next.

\section{Quantitative Analysis of Eye Movement Behaviour}

A visual examination of individual recordings seems to confirm the findings of earlier researchers (Tosi et al., 1997; Goldstein et al., 2007) in that fixations were largely distributed around the centre of a scene and predominately avoided the extremities of the frame. However, an aggregation of the eye movements of several participants indicated a more complex distribution pattern during the first part of a scene (in Ernie's restaurant) compared with patterns observed towards the end of the clip.

A review of the current literature failed to produce any satisfactory metrics that could be deployed. Several researchers have developed measures for static scenes (See Webb \& Renshaw (2008) for a useful review). However, such measures are dependent upon the spatial relationship between static objects of interest being known. The challenge was to devise a metric that would be suitable for dynamic scenes and enable a quantifiable assessment of any observed variations in viewers' behaviour.

Intuitively it seemed probable that any metric developed would comprise two factors: first, the proportion of people, out of the total number of participants viewing the film, fixating within a given time span; secondly the proportion of the fixations that are within a defined locus during the same time period. We called this measure commonality as it is a measure of how many people were looking in the same area at the same moment.

\section{1 'Commonality'}

We divided the screen into an array of two-centimetre ( 56 x 56 pixels) cells. By reading the fixations based on the eye-tracker $\mathrm{X}$ and $\mathrm{Y}$ coordinates, the number of fixations in each cell could be determined. Commonality could now be defined, for any given frame, as the ratio between the total number of potential viewers fixating (as a proportion of the total number of viewers) divided by the number of cells (as a proportion of the total number of cells):

\section{The proportion of viewers fixating in a specific $\mathrm{c} \epsilon$}

The proportion of virtual cells containing fixations

For example, if in a particular frame 10 people's fixations from a possible pool of 60 people were recorded, and eight cells (out of 100) had at least one fixation each, the commonality factor for that frame would be:

$$
\frac{(10 / 60)}{(8 / 100)}=2.08
$$


The commonality factor affords a quantitative measure of how viewers view dynamic scenes over time. To demonstrate this, values of commonality for each frame within each of the clips were calculated and plotted. To save space only the plot of the commonality by frame for the 'Restaurant' scene is shown below in Graph 1. Data from this clip was selected because in the first half of the clip the camera, using a wide angle setting, pans across a crowded restaurant scene, stops (about half way through the clip) and gently zooms in on a specific couple seated at a table centre screen. From this point on the predominating shooting style is of shallow static shots of faces until the main characters leave the restaurant and the clip ends. Graph 1 shows that in the first half of the clip the commonality decreases to a minimum then increases from about halfway through the clip.

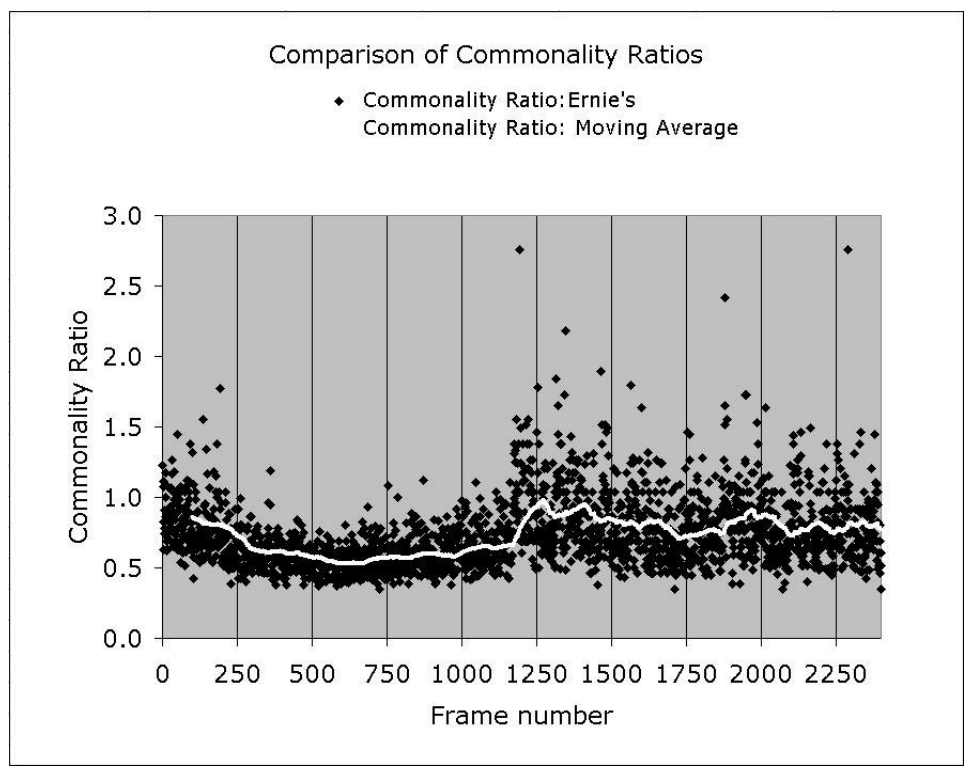

Graph 1: 'Restaurant' scene; frame-by-frame values of commonality for 238 participants.

The continuous (white) line, based on an arbitrarily chosen 20 frame moving average, has been superimposed over the data to assist in the perception of changes in levels of commonality. The change in eye movement behaviour seems to coincide with a change in the director's shooting strategy.

The next step was to determine whether the observed increase in the levels of commonality discernible in Graph 1 could be assessed for statistical significance. In attempting to do this, however, the data exhibited characteristics which rendered its processing from a statistical point of view problematic:

- The allocation of fixations to cells is an arbitrary binning process; the size of the cells could influence the statistics.

- The population of fixations in each cell were surprisingly small. The methodology had afforded a means of capturing the eye movements of 238 people yet the maximum number of fixations attributed to a frame never exceeded 25 . The usu- 
al test for the normality of the calculated data failed to indicate that the locations of these fixations followed a normal distribution in either the $\mathrm{X}$ or $\mathrm{Y}$ plane.

- There was no way of ensuring that the results by frame throughout the film were independent, as individuals' fixations were highly likely to have been included several times in the computation of any variance statistic.

- Some frames might have several clusters of cells with identical fixation frequency but their differentiation from frames with a wider or narrower dispersal of clusters of fixations was difficult to determine. It was important to do this because, intuitively, clusters in close proximity should have higher commonality factor values than widely dispersed but similar cluster frequencies.

The hunt for an explanation as to the possible reasons for the low numbers of fixations in any one cell also gave a clue to solving the other issues listed above. Part of the explanation for the low fixation frequency could be accounted for by the fact that the system aggregates eye gaze positions into fixations - provided certain time and location parameters are met. However, if the eye is on the move, scanning from one part of the screen to the other, for example, then fewer fixations are recorded because the gaze positions are changing too rapidly for aggregation to be permitted. We concluded that whilst viewing a film, as entertainment, audiences tend to scan or follow moving objects in the scene more frequently than they fixate on a particular point on the screen, unless encouraged to do so by the director's shooting strategy.

Another contributory factor may also be the way the eye-tracking software attributes a time stamp to fixations, effectively only counting a fixation when it first occurs.

After reflection it was thought that, by ignoring the fixations as recorded by the eye-tracker software, and reverting to raw eye gaze data in the form of X, Y coordinates of the recorded eye gaze positions, many of these issues might be resolved. This process is described next.

\subsection{A New Approach: Eye Gaze Distribution Analysis}

Unfortunately not all the data saved from the exhibitions was saved at the level of the $\mathrm{X}, \mathrm{Y}$ coordinates of the recorded eye gaze positions and consequently the following analysis is based on the available valid eye gaze data from 163 participants as they watched the 'Restaurant' scene.

It is reasonable to assume that times of high 'commonality' would be indicated for each moment in time - by a decrease in the variation of individuals' eye gazes as all participants would be looking approximately in the same place. Conversely periods of low commonality would have wider variations in $\mathrm{X}$ and $\mathrm{Y}$ coordinates.

Variations in the distribution of eye gaze locations can be measured by calculating the standard deviation of both the $\mathrm{X}$ and $\mathrm{Y}$ coordinates. If these standard deviations are simply multiplied together the product is a measure of the area of gaze.

It is also valid to assume that a simple $t$-test would show the statistical significance of the differences in behaviour between any two parts of the film. A t-test assesses whether two averages - each taken from a set of values which are to be compared are meaningfully different from each other. It requires that the sets of values be normally distributed and so a standard statistical transformation was applied to remove the positive skew which we found in the values for both parts of the film. 


\subsubsection{Applying the Analysis Method}

By analysing the $\mathrm{X}$ and $\mathrm{Y}$ coordinates of the captured eye gaze positions it was possible to determine whether viewing behaviour varied between the two halves of the Ernie's restaurant clip. The t-test showed there to be a significant difference in viewing behaviour between the two parts of the film. In fact the area of variability of the gazes in the first half of the film was estimated to be between 1.7 and 1.9 times the variability in the second half of the film, at the $95 \%$ confidence level.

Given the film Vertigo's key role in the debates on gender in cinema (Mulvey, 1975) we have also applied the statistical measure to see if there are any gender related variations in what is attended to within the scenes. To this end the X, Y standard deviation data was further split according to the gender of the participants. A twosample t-test on this data indicated no clear difference between the two groups and thus no good evidence was found of a gender effect.

Having trialled the use of fixation data, we concluded that for a fair and valid analysis of differences of behaviour on a frame by frame basis it is instead necessary to go back to the 'raw' eye gaze data in order to gain some insight into the way eye movements are influenced by directorial techniques.

From our evidence and that of Goldstein et al (2007) eye gazes are not always at the centre of the scene. They show a good degree of variability in and around it, particularly when the camera is used to pan around a scene. However the way the equipment was set up in this study can hardly be claimed to represent a typical cinematographic experience. We would be cautious about inferring a translation of our results to the big screen until further research has been carried out.

Nevertheless, we have shown how the eye movements of several hundred viewers of a film can be combined into one aggregated visualization that illustrates their collective visual experience. We have also demonstrated that it is possible to test variations in eye movement patterns for statistical significance, opening the way to objective assessments of the director's skill in influencing how people watch films. Our approach can give the creators of dynamic media such as films a new perspective on how their work is received. It also has the potential to generate new, audiencegenerated works of art, over and above the art within the film (Versterby et al., 2005).

\section{References}

Allen, R. C. (1990). From Exhibition to Reception: Reflections on the Audience in Film History. Screen, 31 (4), 347-356.

Bazin, A. (1967). What is Cinema?. California: University of California Press.

Boorstin, J. (1991). The Hollywood Eye: What Makes Movies Work. Boston: Focal Press

Bordwell, D. \& Thompson, K. (2007). Film Art : An Introduction. London: McGraw-Hill Companies.

Clearview Version 2.7.1 for Windows XP (2006). [Computer software]. Vancouver: TOBII.

Coan, J. A., \& Allen, J. J. B. (2007). Handbook of Emotion Elicitation and Assessment. Oxford: Oxford University Press. 
Cutting, J. E. (2005). Perceiving Scenes in Film and in the World. In Anderson, J. D. \& Fisher, B. (Eds.). Motion Picture Theory: Ecological Considerations. Carbondale: Southern Illinois University Press.

D'Ydewalle, G., \& Vanderbeeken, M. (1990). Perceptual and Cognitive Processing of Editing Rules in Film. In: Groner, R., d'Ydewalle, G. \& Parham, R. (Eds.). From Eye to Mind: Information Acquisition in Perception, Search and Reading. Amsterdam: Elsevier.

Duchowski, A. (2007). Eye-tracking Methodology: Theory and Practice. (2 ${ }^{\text {nd }}$ ed.). New York: Springer-Verlag.

Dymtryk, E. (1984). On Film Editing. Boston: Focal Press.

Findlay, J. M. (1984). Properties of the Saccadic Eye Movement System: Introduction. In Gale, A. G., \& Johnson, F. (Eds). Theoretical and applied aspects of eye movement research. Amsterdam: Elsevier.

Goldberg, H. J. \& Wichansky, A. M. (2003). Eye-tracking in Usability Evaluation: A Practitioners Guide. In Hyona, J., Radach, R. \& Deubel, H. (Eds.) The Mind's Eye: Cognitive and Applied Aspects of Eye Movement Research. Amsterdam: Elsevier.

Goldstein, R. B., Woods, R. L. \& Peli, E. (2007). Where People Look When Watching Movies: Do all Viewers Look at the Same Place?. Computers in Biology and Medicine, 37 (7), 957-964.

Haeffner, N. (2005). Alfred Hitchcock. Essex: Pearson.

Henderson, J. M. \& Hollingworth, A. (1998). Eye Movements during Scene Viewing: An Overview. In G. Underwood (Ed.). Eye Guidance in Reading and Scene Perception (pp. 269-293). Oxford: Elsevier.

Hitchcock, A. (Director). (1958). Vertigo [Motion picture]. United States: Paramount Pictures.

Katz, S. (1991). Film Directing: Shot by Shot: Visualizing from Concept to Screen. California: Michael Wiese Productions.

Kohn, B. (2000). Hitchcock at Work. London: Phaidon.

Lane, C., Cryer, T., Che-Guevara, J., Haeffner, N., Raybould, D., Robinson, A., Spiropoulou, S., Stevens, R. (2007). Repossessed Exhibition Catalogue. London Metropolitan University.

Itti, L. (2004). Automatic Attention-Based Prioritiztion of Unconstrained Video for Compression. In SPIE Human Vision and Electronic Imaging: Conference 9 (pp. 272-283). San Jose: ETATS-UNIS.

MaxMSP Version 5.0.5 for Mac OSX (2008). [Computer software]. San Francisco: Cycling74

Mulvey, L. (1975). Visual Pleasure and Narrative Cinema. Screen 16.3 Autumn.

Pepperman, R. D. (2004). The Eye is Quicker: Film Editing: Making a Good Film Better. California: Michael Wiese Productions.

Privitera, C. M., \& Stark, L. W. (2000). Algorithms for Defining Visual Regions-of-Interest: Comparison with Eye Fixations. IEEE Transactions on Pattern Analysis and Machine Intelligence. 22 (9), 970-982.

Rayner, K. (1998). Eye Movements in Reading and Information Processing: 20 Years of Research. Psychological Bulletin, 124 (3), 372-422.

Smith, T.J. (2005). An Attentional Theory of Continuity Editing. Unpublished doctoral dissertation, Edinburgh University.

Spoto, D. (1983). The Dark Side of Genius: the Life of Alfred Hitchcock. London: Frederick Muller.

Staiger, J. (1992). Interpreting Films. Princeton: Princeton University Press.

Stelmach, L., Tam, W. J. \& Hearty, P. (1991). Static and Dynamic Spatial Resolution in Image Coding: An Investigation of Eye Movements. Human Vision, Visual Processing and Digital Display II. 1453, 147-152.

Tobii. (2006). Tobii Technology AB [Computer hardware]. Vancouver.

Tosi, V., Mecacci, L. \& Pasquali, E. (1997). Scanning Eye Movements Made When Viewing Film: Preliminary Observations. International Journal of Neuroscience. 92, 47-52. 
Truffaut, F. (1984). Hitchcock: A Definitive Study of Alfred Hitchcock. (rev. ed.). New York: Simon and Schuster.

Vesterby, T., Voss, J. A., Hansen, J. P., Glenstrup, A. J., Hansen, D. W., \& Rudolph, M. (2005). Gaze-Guided Viewing of Interactive Movies. Digital Creativity, 16 (4), 193-204.

Webb, N. \& Renshaw, A. J. (2008). Eye-tracking in HCI. In Cairns, P., \& A.Cox (Eds.). Research methods for Human-Computer Interaction. Cambridge: Cambridge University Press.

Wooding, D. S. (2002). Fixation Maps: Quantifying Eye-Movement Traces. In Proceedings of the 2002 Symposium on Eye-tracking Research \& Applications. (pp 31-36), New York: ACM. 